\title{
Visibility anomaly of GNSS satellite and support from regional systems
}

\author{
Somnath Mahato ${ }^{1}$, Atanu Santra ${ }^{1}$, Sukabya Dan ${ }^{1}$, Pratibha Verma ${ }^{2}$, \\ P. Banerjee ${ }^{1}$ and Anindya Bose ${ }^{1, *}$ \\ ${ }^{1}$ Department of Physics, The University of Burdwan, Golapbag, Burdwan 713 104, India \\ ${ }^{2}$ Department of Electronics and Communication, National Institute of Technology, Tadepalligudem 534101 , India
}

In a multi-GNSS (global navigation satellite system) environment with operational GPS, GLONASS and Galileo Satellites, the Asia-Oceania region is expected to get better benefits of a large number of GNSS satellites for use. However, it is witnessed that during some parts of the day, no GNSS satellite is present above $60^{\circ}$ elevation angle from many parts of the earth, including India. Real-time data from India show the regular absence of usable GPS satellites above $60^{\circ}$ elevation angles during some parts of the day; addition of GLONASS and Galileo satellites does not improve the situation much. From Burdwan, West Bengal, India at least twice a day, no GNSS satellite is found above $60^{\circ}$ elevation angles for more than $30 \mathrm{~min}$. Simulation study for scattered places of India and data from IGS Centres confirm similar observations, except for the extreme northern region. The global scenario also supports these observations, while the individual operator's country is free from the problem using their own navigational system. The consequences of the problem affect GNSS-based solutions; for locations with obstruction of GNSS signals from low elevation angles, the concurrent occurrence of this incidence poses a threat for seamless GNSS-based navigation through intermittent loss of solution and degraded solution quality. Regional navigation satellite systems (RNSS) help mitigate this problem within the respective service regions. For a large part of the globe, the problem may be allayed using GNSS-RNSS hybrid operation. The result would be important for location-specific GNSS mission planning in strategic, life and safety-critical applications.

Keywords: GNSS, regional systems, satellites, visibility anomaly.

MuLTI-global navigation satellite system (GNSS) has gained popularity with the fully operational global constellations - GPS, GLONASS and Galileo, that support redundant global availability of a large number of satellites $^{1,2}$. Especially for the Asia-Oceania region, there is better availability of multi-GNSS signals in comparison to other parts of the globe, and the advantages and poten-

*For correspondence. (e-mail: abose@phys.buruniv.ac.in) tials of multi-GNSS have been explored by several researchers ${ }^{3-6}$. However, even with a large number of available GNSS satellites, they are not always favourably distributed over the sky for use. It is witnessed that during some parts of the day, all GNSS satellites do not have sufficiently high elevation angles ${ }^{7-9}$. Under the open sky or in aviation applications, this issue does not impact the position solution. Nevertheless, for constrained visibility environments like urban areas with high-rise buildings or forest environments, signals from the lower elevation angles are obstructed and during these periods there may not be enough satellites from high elevation angles. The combined situation may result in a loss of solution or degraded solution quality. Therefore, it is interesting to study the time-specific problem of non-availability of GNSS satellites and the possible mitigation methods. Here, the objectives are to study: (i) the unavailability of GNSS satellites above $60^{\circ}$ elevation angles; (ii) the situation from different parts of India and the world through simulations; (iii) the effects of this situation on solution quality while operating within a constrained visibility location, and (iv) contribution of the regional satellite navigation systems (RNSS) to mitigate the situation.

Study on the unavailability of GNSS satellites above $60^{\circ}$ elevation angle

\section{Primary observation from GNSS Laboratory, Burdwan (GLB), India}

Primary observations are found using multi-GNSS data in the 'open sky' condition; absence of GPS satellites above $60^{\circ}$ elevation angles for some parts of the day was noted during 2015-16 (ref. 10). A subsequent detailed study showed repetition of the situation even in the presence of a large number of GPS/GNSS satellites above $10^{\circ}$ elevation angles; such typical examples are shown in Figure $1 a$ and $b$ for GPS and GPS + GLONASS + Galileo respectively. It is seen that addition of GLONASS and Galileo constellations does not always improve the situation much, and there are periods when no satellite is found for use above $60^{\circ}$ elevation angle, which is taken as the lower boundary of high elevation angle. For the case 
Table 1. Observation of low visibility of satellites from GNSS Laboratory, Burdwan (GLB), India

\begin{tabular}{|c|c|c|c|c|c|}
\hline \multirow{2}{*}{$\begin{array}{l}\text { Date } \\
01 / 06 / 2017\end{array}$} & Constellation & \multicolumn{3}{|c|}{$\begin{array}{l}\text { Time span for maximum } \\
\text { elevation }<60^{\circ} \text { (hours IST) }\end{array}$} & \multirow{2}{*}{$\begin{array}{c}\begin{array}{c}\text { Duration } \\
(T ; \min )\end{array} \\
47\end{array}$} \\
\hline & \multirow[t]{4}{*}{ GPS } & $01: 16: 18$ & - & 02:04:03 & \\
\hline & & $13: 50: 03$ & - & $14: 32: 18$ & 42 \\
\hline & & $16: 29: 48$ & - & $17: 44: 03$ & 74 \\
\hline & & $22: 49: 48$ & - & 23:08:03 & 18 \\
\hline & \multirow[t]{5}{*}{ GLONASS } & 00:00:00* & - & $00: 05: 03$ & 05 \\
\hline & & $00: 22: 33$ & - & $02: 51: 18$ & 149 \\
\hline & & $08: 13: 18$ & - & $10: 46: 33$ & 153 \\
\hline & & $15: 26: 33$ & - & $15: 50: 18$ & 23 \\
\hline & & $16: 23: 48$ & - & $18: 52: 33$ & 148 \\
\hline & \multirow[t]{4}{*}{ Galileo } & 00:00:00* & - & $05: 10: 45$ & 310 \\
\hline & & $08: 13: 33$ & - & $09: 23: 18$ & 55 \\
\hline & & $09: 55: 45$ & - & $01: 20: 42$ & 206 \\
\hline & & $16: 39: 33$ & - & $18: 49: 33$ & 130 \\
\hline & \multirow[t]{2}{*}{ GPS + GLONASS + Galileo } & $01: 16: 18$ & - & 02:04:03 & 47 \\
\hline & & $16: 39: 33$ & - & $17: 44: 03$ & 64 \\
\hline \multirow[t]{2}{*}{$02 / 06 / 2017$} & & $01: 12: 18$ & - & 01:59:48 & 47 \\
\hline & & $16: 35: 48$ & - & $17: 40: 03$ & 64 \\
\hline
\end{tabular}

*Below $60^{\circ}$ starts from the previous day.

Table 2. Representative samples for the absence of GNSS satellites above $60^{\circ}$ elevation angles in hybrid operation using Orbitron (simulation Location: GLB, India)

\begin{tabular}{lccccc}
\hline Date & \multicolumn{2}{c}{$\begin{array}{c}\text { Period with maximum elevation }<60^{\circ} \text { for } \\
\text { GPS + GLONASS + Galileo hybrid operation }\end{array}$} & $\begin{array}{c}\text { Duration } \\
(T ; \text { min) }\end{array}$ & $\begin{array}{c}\text { Maximum elevation of all visible } \\
\text { satellites during } T \text { (degrees) }\end{array}$ \\
\hline $02 / 05 / 2017$ & $03: 21: 09$ & - & $04: 08: 24$ & 47 & 59 \\
$02 / 05 / 2017$ & $18: 39: 05$ & - & $19: 49: 21$ & 70 & 47 \\
$04 / 05 / 2017$ & $03: 12: 54$ & - & $03: 59: 54$ & 77 \\
$04 / 05 / 2017$ & $18: 23: 33$ & - & $19: 40: 49$ & 48 & \\
$06 / 05 / 2017$ & $03: 04: 34$ & - & $03: 52: 04$ & 76 & \\
$06 / 05 / 2017$ & $18: 15: 20$ & - & $19: 31: 56$ & & \\
\hline
\end{tabular}

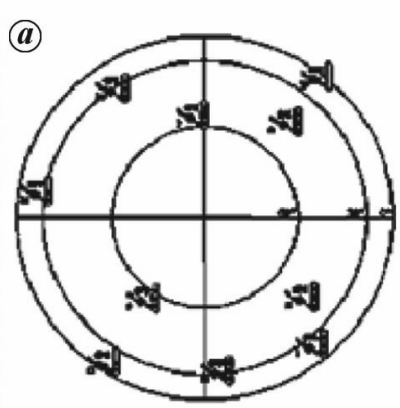

8 February 2017 (GPS)

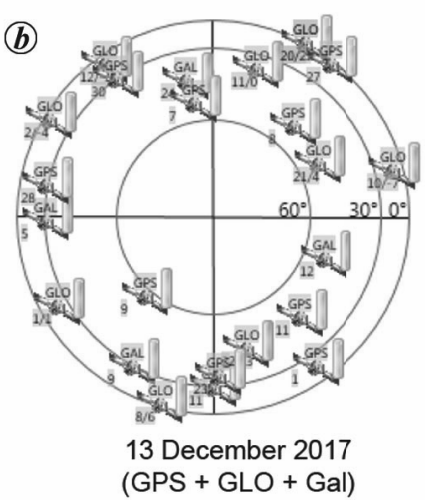

$(\mathrm{GPS}+\mathrm{GLO}+\mathrm{Gal})$
Figure 1. Typical 'skyplots' for all tracked satellites below $60^{\circ}$ elevation angles from GLB, India for (a) GPS and (b) GPS + GLONASS + Galileo. For $(\boldsymbol{b}), 20$ GNSS satellites are present above $10^{\circ}$ elevation. This situation occurs twice a day for $>20$ min duration.

presented in Figure $1 b$, even in presence of 8 GPS, 7 GLONASS, 5 Galileo, none is found above $60^{\circ}$ elevation.

To systematically analyse the situation, maximum elevation angles of satellites from individual and hybrid operation are studied. The periods ( $T$, say) for which the elevation angles of all the satellites are $<60^{\circ}$ are presented in Tables 1 and 2 for $T>10 \mathrm{~min}$. It is noted that for the individual constellation, the problem of "no satellite above $60^{\circ}$, occurs several times over the day with increasing durations for GPS, GLONASS and Galileo satellites respectively.

Now, even in tri-constellation hybrid operation, complete absence of GNSS satellite above $60^{\circ}$ elevation angles occurs if such condition arises concurrently for each of the individual constellations. The situation leads to an unfavourable condition for seamless navigation. However, a hybrid operation helps to complement the visibility of individual constellations. For hybrid multiGNSS constellation, non-availability of any GNSS satellite above $60^{\circ}$ elevation angle over a long period from GLB, India may be seen from the data in Tables 1 and 2 during May and June 2017; the situation is witnessed twice a day with periods of more than $30 \mathrm{~min}$.

To visually inspect the situation, results for 1-3 June 2017 are shown in Figure 2 where the variation of maximum elevation angles of individual and hybrid constellation shows systematic repetition of 'no GNSS above $60^{\circ}$ '. 


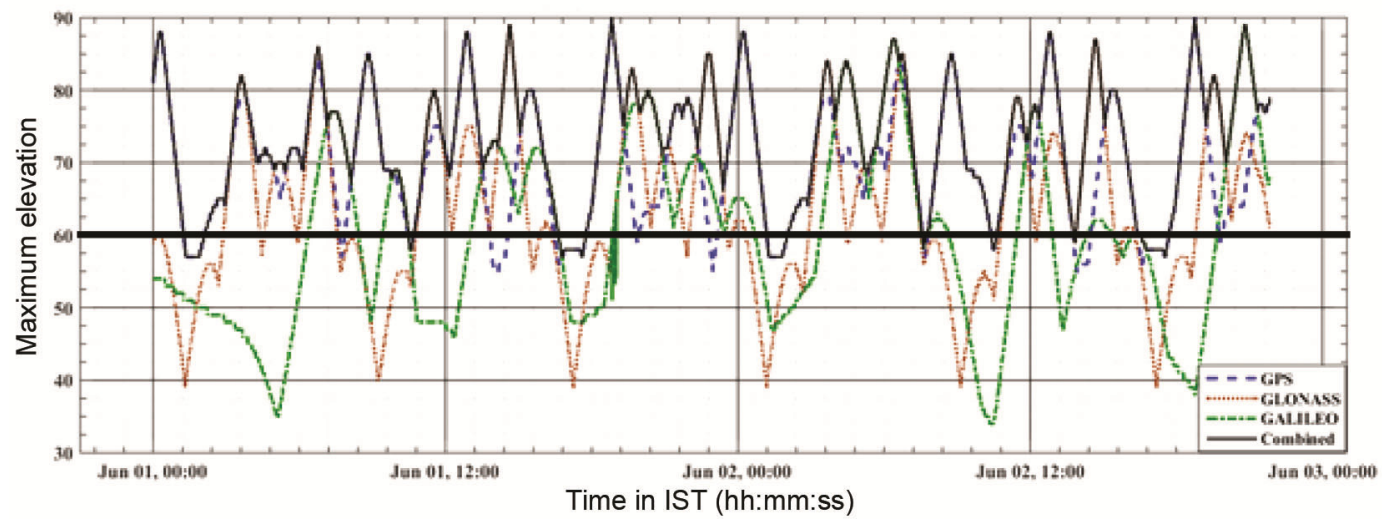

Figure 2. Variation of maximum elevation of satellites in standalone and hybrid GNSS operation observed during June 2017 from GLB, India. The thick black line corresponds to $60^{\circ}$ elevation angle.

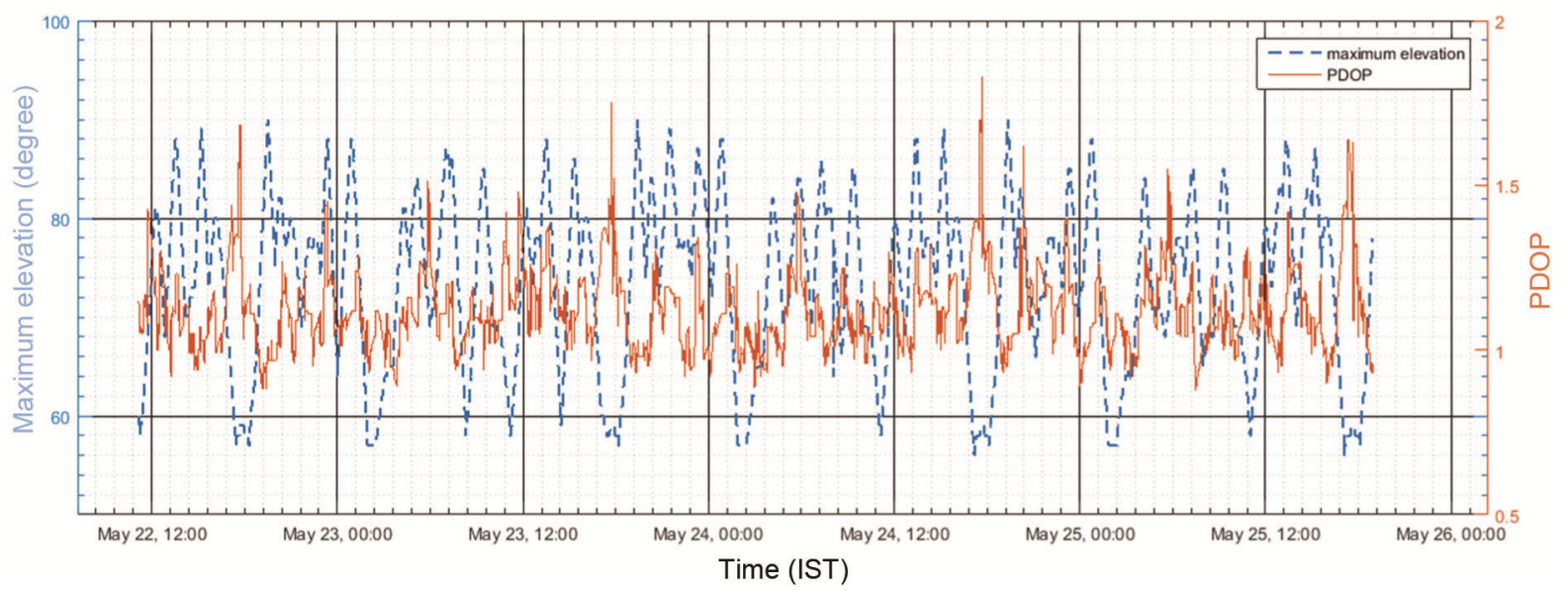

Figure 3. Variation of maximum elevation of satellites and satellite geometry, 22-26 May 2018, GLB, India.

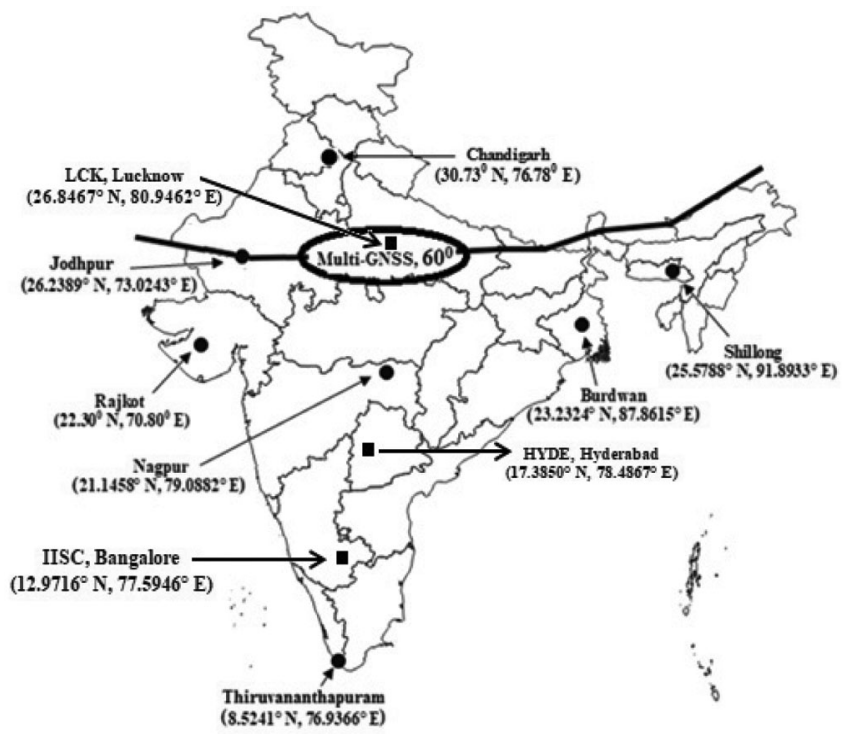

Figure 4. Contour (dark line marked with 'Multi-GNSS $60^{\circ}$ ') below which the problem of non-availability of GNSS satellites from $>60^{\circ}$ elevation angles exists during some parts of the day in India. The squares show IGS station locations ${ }^{14}$.
In many cases, the hybrid operation maximum elevation curve follows the GPS curve, pointing towards better visibility of GPS satellites from the region vis-à-vis the other two constellations. Figure 3 shows variation of maximum elevation of hybrid constellation satellites and satellite geometry during May 2018 from GLB, India. The long-term (2015-2018) observation establishes the phenomenon to be a general one and indicates higher Precision Dilution of Position (PDOP) values during the condition.

\section{Simulated observations for GNSS visibility from India}

Using Orbitron, a popular simulation software ${ }^{11}$, GNSS satellite visibility from GLB, India was calculated using the latest two-line element (TLE) files obtained from the CelesTrack website ${ }^{12}$. The results show the situation of non-availability of GNSS satellites above $60^{\circ}$ elevation angles for more than $30 \mathrm{~min}$ twice a day from GLB, India. The simulated results agree well with the real-time data and results presented in Table 1 and Figure 2. 
Table 3. Absence of GNSS satellites above $60^{\circ}$ elevation angles in tri-constellation hybrid operation (simulation using Orbitron)

\begin{tabular}{lccccc}
\hline \multirow{2}{*}{ Observation location } & Date & $\begin{array}{c}\text { Time with max elevation is less than } 60^{\circ} \\
(T) \text { (hours IST) for hybrid operation }\end{array}$ & $\begin{array}{c}\text { Duration } \\
(T ; \text { min) }\end{array}$ & $\begin{array}{c}\text { Maximum elevation of all tracked } \\
\text { satellites during } T(\text { degree) }\end{array}$ \\
\hline Thiruvananthapuram & $16 / 05 / 2017$ & $02: 40: 13$ & - & $03: 10: 40$ & 31 \\
& $12 / 12 / 2017$ & $12: 03: 00$ & - & $12: 53: 00$ & 50 \\
& $24 / 04 / 2018$ & $03: 13: 00$ & - & $03: 44: 00$ & 31 \\
Nagpur & $16 / 05 / 2017$ & $02: 50: 17$ & - & $03: 41: 07$ & 51 \\
& $12 / 12 / 2017$ & $12: 33: 00$ & - & $13: 24: 00$ & 51 \\
Rajkot & $24 / 04 / 2018$ & $03: 25: 00$ & - & $04: 14: 00$ & 49 \\
& $16 / 05 / 2017$ & $11: 49: 16$ & - & $12: 53: 25$ & 64 \\
Shillong & $12 / 12 / 2017$ & $21: 28: 00$ & - & $22: 11: 00$ & 43 \\
& $24 / 04 / 2018$ & $12: 24: 00$ & - & $13: 19: 00$ & 54 \\
Jodhpur & $16 / 05 / 2017$ & $17: 43: 14$ & - & $19: 01: 25$ & 78 \\
& $12 / 12 / 2017$ & $03: 38: 00$ & - & $04: 45: 00$ & 67 \\
& $24 / 04 / 2018$ & $18: 19: 00$ & - & $19: 35: 00$ & 76 \\
& $16 / 05 / 2017$ & $11: 40: 00$ & - & $11: 49: 00$ & 09 \\
& $12 / 12 / 2017$ & $21: 18: 00$ & - & $21: 22: 00$ & 04 \\
& $24 / 04 / 2018$ & $12: 15: 00$ & - & $12: 22: 00$ & 08 \\
\hline
\end{tabular}

In our next study on GNSS high-elevation visibility problem over the Indian region, we employed simulated data using Orbitron for six scattered locations of India Thiruvananthapuram, Nagpur, Rajkot, Shillong, Jodhpur and Chandigarh (Figure 4). GPS + GLONASS + Galileo satellite visibility was simulated for few consecutive days during two campaigns separated by nearly seven months; Table 3 shows the results. Absence of all GNSS satellites above $60^{\circ}$ elevation during some parts of the day was observed from five places situated in the middle to southern parts of India. For Chandigarh in northern India, this problem was not witnessed. The interesting observations from Table 3 are: time span of the absence of satellites from high elevation angles increases with the sequential shift of the observation point from south to north and for locations further north of the country, the problem disappears.

Next, simulation was performed using an in-house developed utility that uses GNSS TLE files, System Tool Kit $(S T K)^{13}$ and MatLab. The utility first calculates the satellite orbital positions, which are passed to a Matlab program to finally generate the GNSS satellite visibility patterns for any user-specified location. These results are validated using both real-time data and Orbitrontherefore, either the Orbitron or this utility can be used for generalized GNSS satellite visibility study, and both have been used for the studies presented here.

GPS data from three IGS stations of India, viz. Lucknow (LCK), Hyderabad (HYDE) and Bengaluru (IISc) ${ }^{14}$ were used and satellite elevation angles calculated from the RINEX data using RTKLib ${ }^{15}$. Figure 5 shows the maximum elevation angles of tracked satellites. Real-time data from the IGS stations also support our findings; IISc situated at the southern part of India is prone to the problem for maximum duration, while in the case of LCK the periods of the problem are shorter. Considering the location-dependent nature of the problem, next we analysed its global pattern of occurrence.

\section{GNSS low visibility study over the globe}

To extend the study over the globe, simulated results for global observation points separated by $5^{\circ}$ each in latitude and longitude were found using the in-house developed tool described earlier in the text. Figure 6 shows the locations, where such situations occur for a span of $>20 \mathrm{~min}$ over a day. To validate the global simulated results, IGS GPS data for two locations, viz. Addis Ababa, Ethiopia (ADIS) situated in the affected region, and Quincy, USA (QUIN) situated outside the affected area were used ${ }^{14}$. Representative results are shown in Figure 7 that support the simulated results presented in Figure 6 and our findings.

Therefore, the non-availability of GNSS satellites from high elevation angles during some part of the day is a repetitive, systematic global problem. The phenomenon is location-specific, being prominent around the low- to mid-latitude zones that include India, and both the polar regions (Figure 6). It can be seen from Figure 6 that the scenario of 'unavailability of GPS satellites above $60^{\circ}$ elevation angle' does not occur in most parts of USA. Similarly over Russia 'unavailability of GLONASS satellites above $60^{\circ}$ elevation angle' does not generally occur. This observation is perhaps associated with the constellation design issue, as the affected time shifts in a systematic manner.

The scenario of 'unavailability of GNSS satellites above $60^{\circ}$ elevation angle' occurs for some time each day over many parts of the globe even for an operational triband hybrid multi-GNSS operation. The lower number of available satellites for location-specific conditions and 

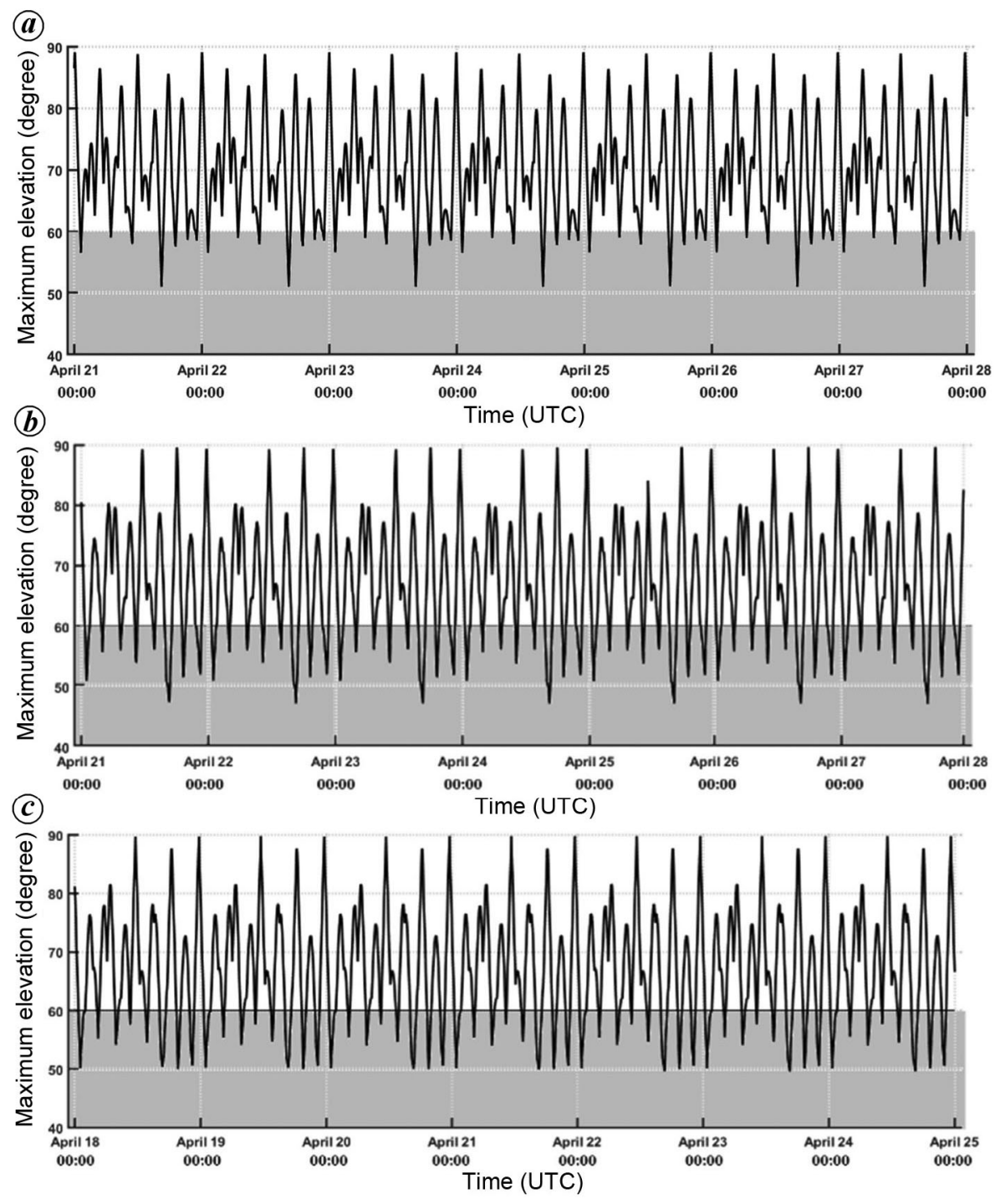

Figure 5. Variation of maximum elevation angle of GPS satellites from IGS stations (a) Lucknow (LCK), (b) Hyderabad (HYDE) and (c) Bengaluru (IISc) India. April 2018.

overall inferior geometry during these situations may affect seamless positioning and degrade the solution quality, as discussed subsequently. Nevertheless, in general, enough GNSS satellites are present all the time, irrespective of elevation issues in the open-sky condition.

\section{Effects of unavailability of GNSS satellites above $60^{\circ}$ elevation angle in navigation}

First, we consider the results of a study from India on the visibility of GNSS satellites in constrained situations ${ }^{16}$. By intentionally masking the elevation angle up to $45^{\circ}$ for the GPS + GLONASS operation, we found that even in the presence of 8-10 satellites in each of the GPS and GLONASS constellations, only 3-4 could be used. Thus, if the GNSS satellite visibility is obstructed from low to mid regions of the sky, a single constellation-based solution may not be possible in many cases. This study was conducted during arbitrary periods without considering the currently discussed GNSS visibility problem. Now let us consider both the location- and time-specific situations together, i.e. a GNSS user is within a location where the satellite visibility is restricted by obstructions (e.g. highrise buildings, foliage) concurrently during the periods of "no GNSS satellite above $60^{\circ}$ elevation'9,17 (e.g. as shown in Table 1). In such situations, satellite visibility from the lower angles is obstructed by locationspecific conditions, and signals from high elevation angles are unavailable due to time- and locationspecific conditions. As a result, seamless navigation is seriously impaired. The situation is particularly relevant for Indian conditions, where buildings or foliage are present on the sides of narrow roads/lanes. Studies were 

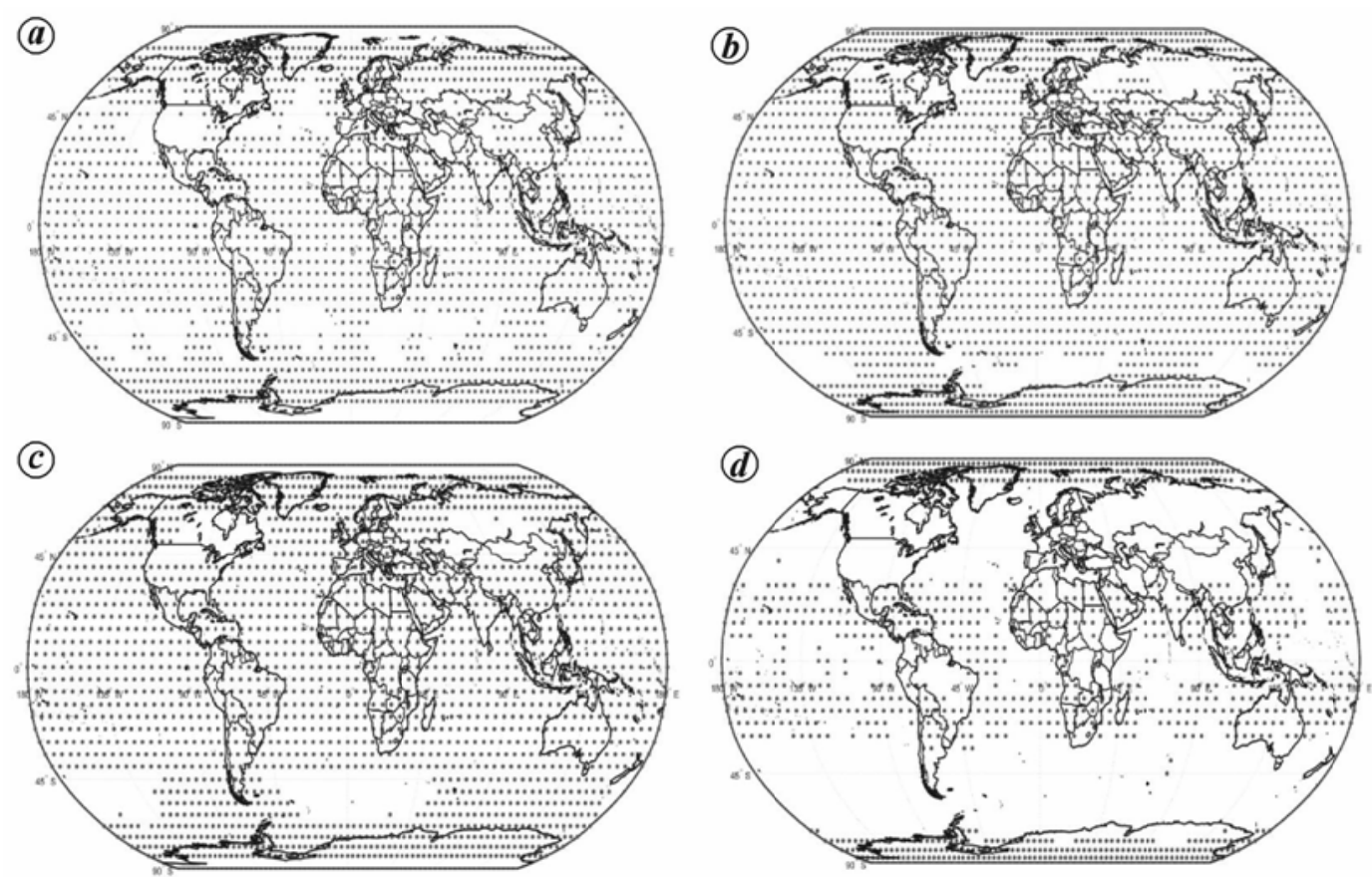

Figure 6. Locations of time-specific GNSS visibility problem over the globe. The dark dots show locations where all (a) GPS, (b) GLONASS, (c) Galileo and (d) GPS + GLONASS + Galileo satellites lie below $60^{\circ}$ elevation angle for more than 20 min over a day; 24 April 2018 and 6 April 2019.
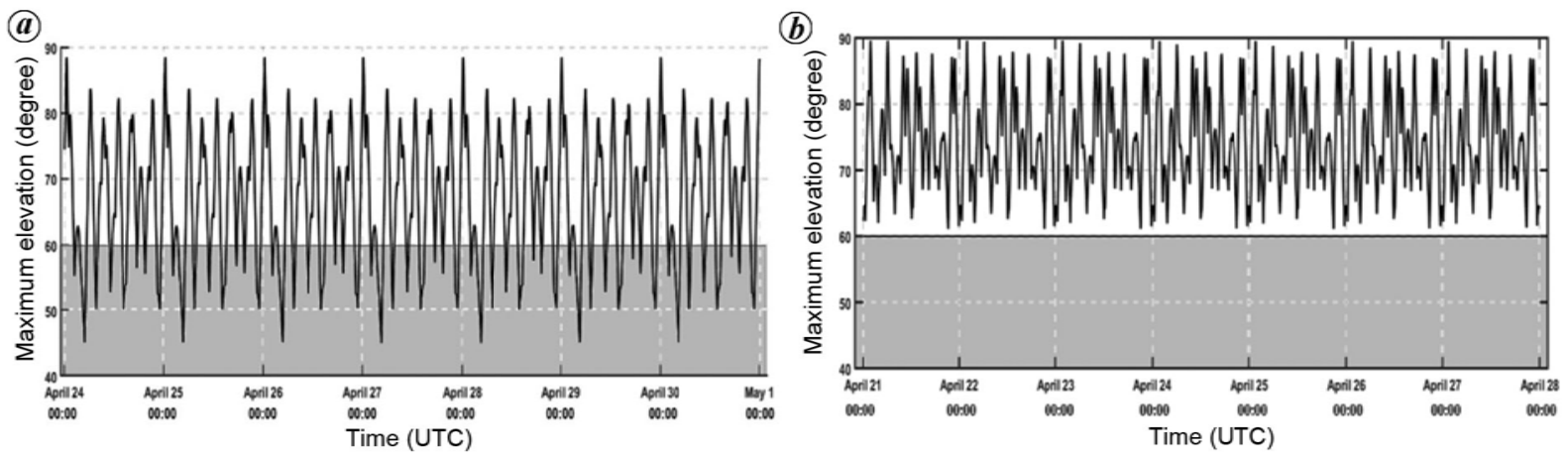

Figure 7. Variation of maximum elevation angle of GPS satellites from IGS station (a) Addis Ababa $\left(8.9806^{\circ} \mathrm{N}, 38.7578^{\circ} \mathrm{E}\right.$, Ethiopia (ADIS), 24 April-1 May 2018 and (b) Quincy (42.2529 N, 71.0023 $\left.{ }^{\circ} \mathrm{W}\right)$, USA (QUIN), 21-28 April 2018.

conducted to observe the effects using real-time experiments.

In the case of one experiment, a car-mounted GNSS receiver moving at $15-20 \mathrm{kmph}$ speed on the roads (4$5 \mathrm{~m}$ wide) of a densely populated area of Kolkata, India, intermittently failed to receive at least four satellite signals, and therefore could not provide position solution during the affected period of "no satellites above $60^{\circ}$, condition $^{9}$. In another case, a motorbike-mounted GNSS receiver moved within an urban canyon region of Burdwan through the same route during the affected period (onward journey) and normal condition (return journey). During the affected period, lower solution success rate was observed than the normal period, and the solution quality had also degraded $^{17}$.
To compare the hybrid GNSS solution quality during the affected and normal periods, a static survey was conducted using a GPS + GLONASS + Galileo survey-grade receiver from a constrained city environment (Figure $8 a$ ). Representative skyplots of the two situations are shown in Figure $8 b$ and $c$ respectively. Position solutions for the affected and normal periods were analysed. Figure $8 d$ and Table 4 show the results. Lower success probability (93.4\% in comparison to $100 \%)$ and inferior solution quality during the affected period of 'no GNSS above $60^{\circ}$ elevation angle' were observed comparing the associated solution quality parameters, distance root mean square (DRMS), circular error probable (CEP), spherical error probable (SEP), mean radial spherical error $(\mathrm{MRSE})^{18}$. 
Table 4. Comparison of position solution in normal and affected periods for static tri-constellation hybrid operation in constrained city environment of Burdwan, India on 14 July 2018

\begin{tabular}{|c|c|c|c|c|c|c|c|c|c|}
\hline \multirow[b]{2}{*}{ Condition (time, IST) } & \multirow{2}{*}{$\begin{array}{c}\text { Total epochs } \\
\text { (average PDOP) }\end{array}$} & \multirow{2}{*}{$\begin{array}{l}\text { Total solution obtained } \\
\text { (solution success) }\end{array}$} & \multicolumn{3}{|c|}{ Maximum variation (m) } & \multirow{2}{*}{$\begin{array}{l}\text { 2DRMS } \\
\qquad(\mathrm{m})\end{array}$} & \multirow{2}{*}{$\begin{array}{l}\text { CEP } \\
(\mathrm{m})\end{array}$} & \multirow{2}{*}{$\begin{array}{l}\text { SEP } \\
(\mathrm{m})\end{array}$} & \multirow{2}{*}{$\begin{array}{l}\text { MRSE } \\
(\mathrm{m})\end{array}$} \\
\hline & & & Latitude & Longitude & Altitude & & & & \\
\hline $\begin{array}{l}\text { Normal }(06: 53-07: 10 \\
\text { and } 08: 24-09: 14)\end{array}$ & $4012(3.05)$ & $4012(100 \%)$ & 41.8 & 5.64 & 26.08 & 11.74 & 4.22 & 5.45 & 6.97 \\
\hline $\begin{array}{l}\text { Affected period } \\
\qquad(07: 10-08: 24)\end{array}$ & $4441(6.23)$ & $4146(93.35 \%)$ & 46.94 & 30.05 & 126.05 & 11.8 & 4.87 & 11.30 & 15.17 \\
\hline
\end{tabular}
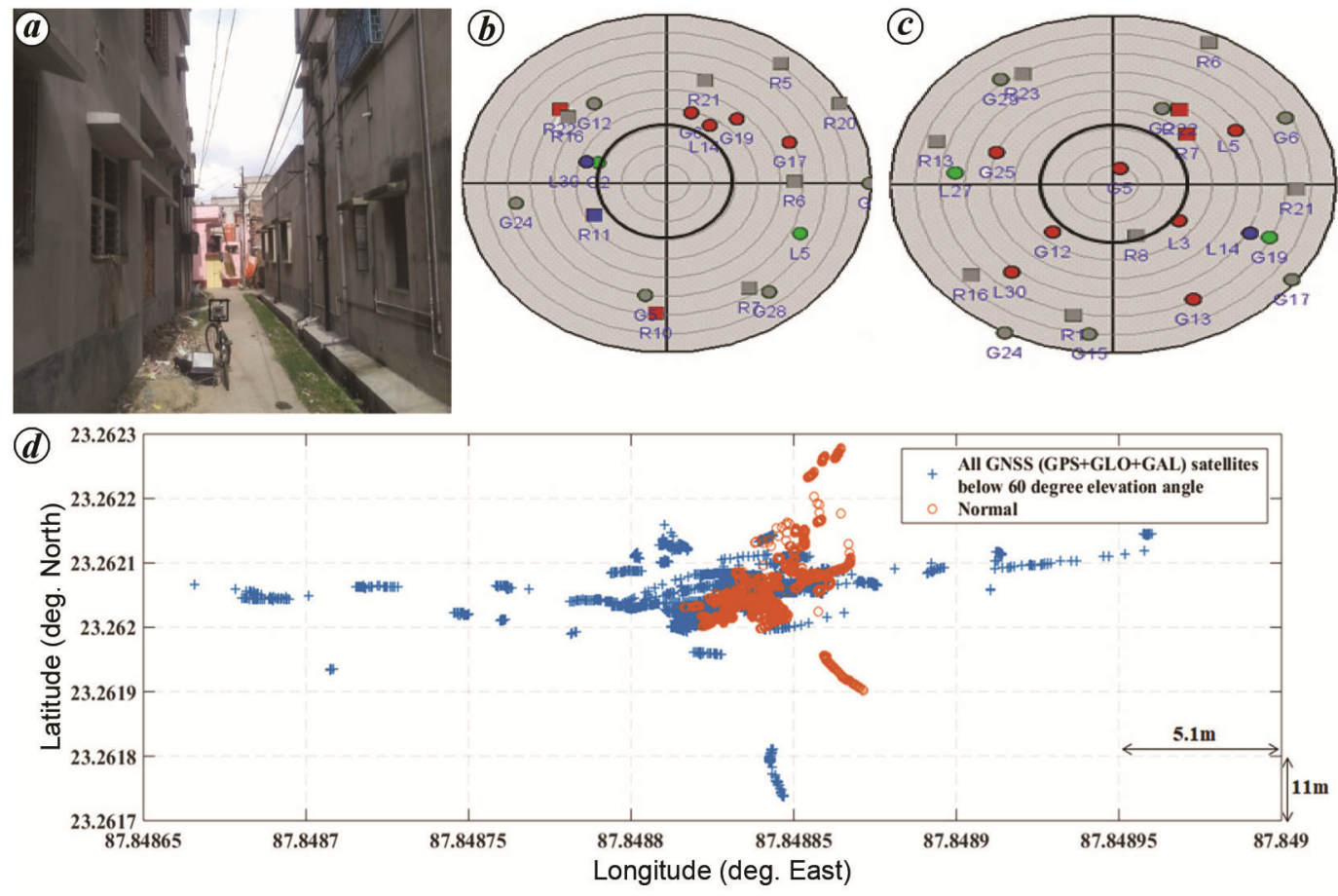

Figure 8. Comparison of solution quality in GPS + GLONASS + Galileo hybrid operation within a constrained city environment of Burdwan, India, 14 July 2018. $\boldsymbol{a}$, Survey location; $\boldsymbol{b}$, Skyplot of the GPS + GLONASS + Galileo hybrid operation with 'no satellite above 60' at 07:27:50 UTC; $c$, Skyplot of the GPS + GLONASS + Galileo hybrid operation during normal condition; the black circle represents $60^{\circ}$ elevation circle; $\boldsymbol{d}$, Comparison of two-dimensional position solutions during affected and normal periods.

These real-time exercises prove the predicted problem of intermittent loss of solutions and degraded solution quality due to location-specific insufficient satellite conditions and the inferior satellite geometry during the periods of 'no GNSS satellites above $60^{\circ}$ elevation angle'.

\section{Benefit of regional navigation satellite systems to mitigate this visibility problem}

NAVigation with Indian Constellation (NavIC) and Quasi Zenith Satellite System (QZSS) are the two operational RNSS from India and Japan ${ }^{19,20}$. This section presents the contribution of the RNSS to mitigate the aforesaid visibility problem of GNSS. Using the method discussed above, the visibility status for GNSS + RNSS satellites was generated (Figures 9 and 10).

From Figures $6 d$ and $9 a$, mitigation of the visibility problem can be witnessed for Japan, its neighbouring regions, and the northern and eastern parts of Australia when QZSS is added with GPS + GLONASS + Galileo; at least one satellite always remains present for use above $60^{\circ}$ elevation angle. Similarly, Figures $6 d$ and $9 b$ suggest mitigation of the problem for India, its surrounding regions, the southern and eastern parts of Africa, and west to the north-western part of Australia when NavIC is added with GPS + GLONASS + Galileo constellations. In Figure 9, all periods of the problem $>20$ min have been added together to show the cumulative time of such events over a day. Now, if both QZSS and NavIC are used together with GPS + GLONASS + Galileo, the 

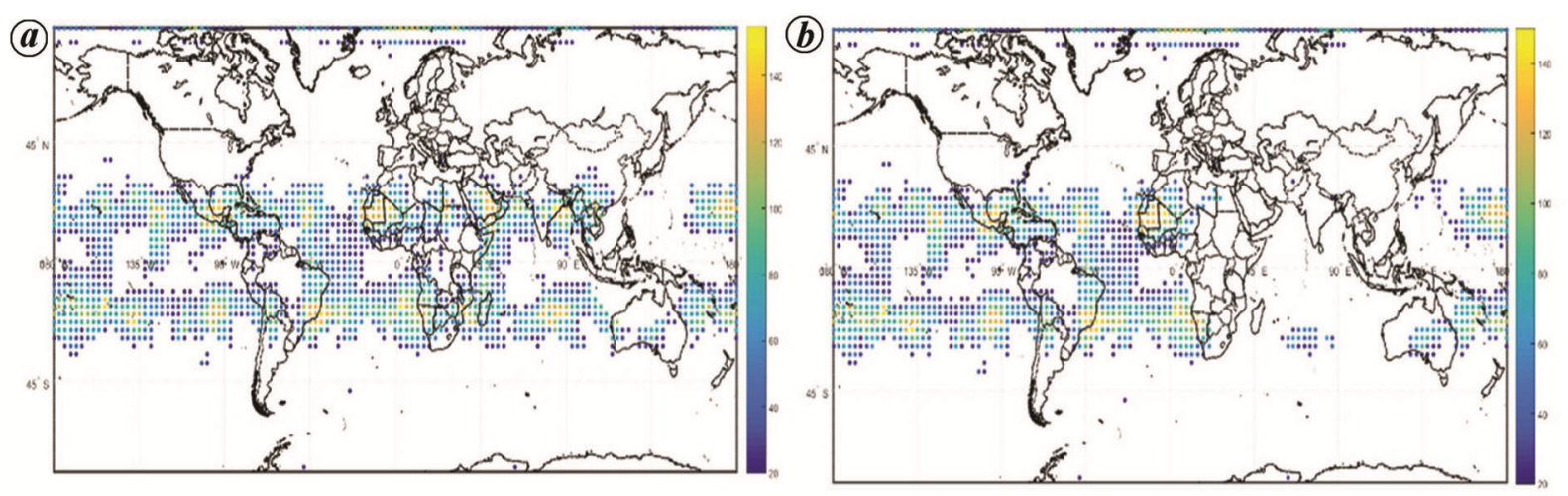

Figure 9. Location of GNSS visibility problem over the globe during some parts of the day, 3 December 2018. The dark dots show locations where $(\boldsymbol{a})$ all GPS + GLONASS + Galileo + QZSS satellites and $(\boldsymbol{b})$ GPS + GLONASS + Galileo + NavIC satellites lie below $60^{\circ}$ elevation angles for $>20$ min over a day. The vertical colour bars show cumulative time span of the problem over the day.

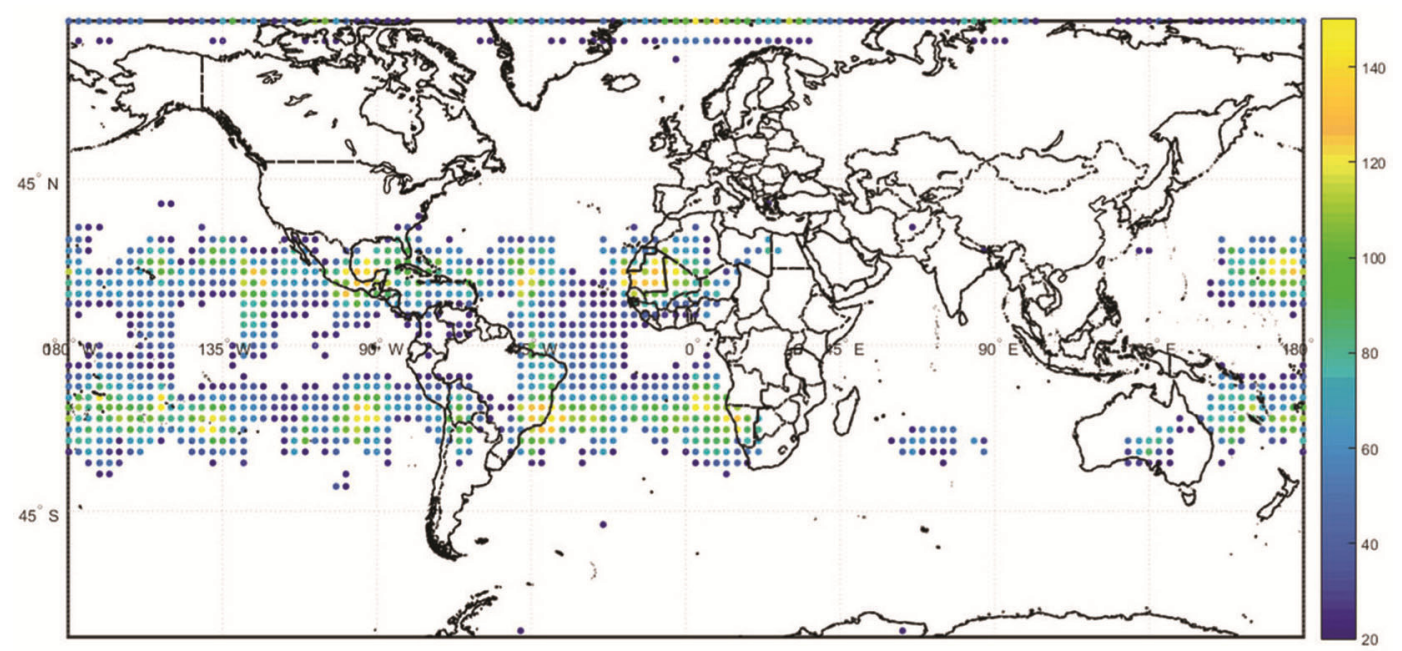

Figure 10. Location of navigation satellite visibility problem over the globe, 3 December 2018. The dark dots show locations where all GPS + GLONASS + Galileo + NavIC + QZSS satellites lie below $60^{\circ}$ elevation angles during some parts of the day. The colour bar on the right shows cumulative time span of the problem over the day.

global scenario is presented in Figure 10. Comparison of Figures 6 and 10 reveals that using the regional navigation systems together with GNSS, the problem of nonavailability of GNSS satellites above $60^{\circ}$ elevation angle is alleviated for a large part of the globe, ensuring seamless and accurate navigation solutions.

\section{Concluding remarks}

This article discusses the location- and time-specific problem of GNSS signal visibility. From real-time observations and simulations, it is found that in the southern to mid-northern parts of India, the problem of GNSS satellite visibility from higher elevation angles exists during some parts of the day. For the extreme northern part and further north of the country, the problem disappears. Through simulation results supported by real-time data, similar global situations are witnessed depending on the location of observation.
The situation does not affect the serviceability of GNSS in open view of the sky, but within degraded visibility conditions like urban canyons, forest roads or deep trenches where the signals from lower elevation angles are simultaneously blocked, this typical problem leads to severe impairment of continuous GNSS-based solution and accuracy, most importantly, for safety-critical applications.

Proper integration of the regional systems - NavIC and QZSS satellites with GPS/GNSS helps improve the situation over the respective regional service systems. With the help of QZSS + NavIC, degradation of satellite visibility within constrained environments can be resolved from the middle to eastern parts of the globe supporting seamless position solution and improved solution quality.

1. Current GNSS, 2017; http://www.gps.gov/systems/gnss/ (accessed on 12 September 2018).

2. European Service Center, 2019; https://www.gsa.europa.eu/about/ what-we-do/services (accessed on 12 January 2019). 
3. Tian, S., Li, G., Chang, J., Li, Y. and Tian, X., Potential benefits of GPS/GLONASS/GALILEO integration in an urban canyon Hong Kong. J. Navig., 2010, 63, 681-693; doi:10.1017/ S0373463310000081.

4. Farah, A., Assessment study of static-PPP convergence behaviour using GPS, GLONASS and mixed GPS/GLONASS observations. Artif. Satellites, 2014, 49(1), 55-61; doi:10.2478/arsa-2014-0005.

5. Choy, S., Zhang, S., Lahaye, F. and Héroux, P., A comparison between GPS-only and combined GPS + GLONASS precise point positioning. J. Spat. Sci., 2013, 58(2), 169-190; doi:10.1080/ 14498596.2013.808164

6. Ryan, S. and Lachapelle, G., Impact of GPS/Galileo integration on marine navigation. In IAIN World Congress/ION Annual Meeting, San Diego, USA, 2000, vol. 721, p. 731.

7. Santra, A., Mahato, S., Ghosh, P., Dan, S. and Bose, A., A study using multi-GNSS services in Indian semi-urban environment. In IETE National Conference on Materials, Devices and Circuits in Communication Technology, Burdwan, 22-23 June 2018, pp. 7780; ISBN 978-81-888391-55-4.

8. Bose, A., Mahato, S., Santra, A., Dan, S. and Verma, P., On time dependent GNSS satellite visibility problem from high elevation angle and NavIC contribution. In Tenth Multi GNSS Asia Conference, Melbourne, Australia, 23-25 October 2018.

9. Santra, A., Mahato, S., Dan, S., Verma, P., Banerjee, P. and Bose, A., Performance analysis of IRNSS-GPS hybrid operation: study in Indian metropolis environment. In Fifth International Conference on Signal Processing and Integrated Networks (SPIN), Noida, 2018, pp. 111-115; doi:10.1109/SPIN.2018.8474183.

10. Mandal, S., Studies on IRNSS and other multi-GNSS and development of GNSS-based cost-effective applications. M. Tech. dissertation, The University of Burdwan, 2016.

11. Stoff, S., Orbitron software, 2005; http://www.stoff.pl (accessed on 17 April 2017).

12. Kelso, T. S., Updated TLEs of Orbitron, 2000; https://www. celestrak.com/NORAD/elements/ (accessed on 2, 4, 6 and 16 May 2017, 1 and 2 June 2017, 12 December 2017, 24 April 2018, 3 November 2018 and 3 December 2018, 6 April 2019).
13. AGI, Systems Tool Kit (STK), 2016; https://www.agi.com/ products /engineering-tools (accessed on 19 December 2017).

14. Silver, M., Scripps Orbit Permanent Array Center-IGS Data Achieve, 2002; http://garner.ucsd.edu/ (accessed on 5 April 2018).

15. Takasu, T., RTKLIB, 2006; http:/github.com/tomojitakasu/ RTKLIB (accessed on 11 January 2017).

16. Sarkar, S. and Bose, A., Studies on solution accuracy of GLONASS from India. Gyrosc. Navig., 2016, 7(1), 39-49; doi:10.1134/S2075108716010119.

17. Santra, A., Mahato, S., Mandal, S., Dan, S., Verma, P., Banerjee, P. and Bose, A., Augmentation of GNSS utility by IRNSS/NavIC constellation over the Indian region. Adv. Space Res., 2019, 63(9), 2995-3008; doi:10.1016/j.asr.2018.04.020.

18. Santra, A., Mahato, S., Dan, S. and Bose, A., Precision of satellite-based navigation position solution: a review using NavIC data. J. Inf. Optim. Sci., 2019, 40(8), 1683-1691; doi:10.1080/ 02522667.2019.1703264.

19. Dan, S., Santra, A., Mahato, S. and Bose, A., NavIC performance over the service region: availability and solution quality. Sadhana, 2020, 45, doi:10.1007/s12046-020-01375-5.

20. GPS World, Japan's QZSS service now officially available. 2018; https:/www.gpsworld.com/japans-qzss-service-now-officiallyavailable/ (accessed on 3 December 2018).

ACKNOWLEDGEMENTS. We thank the Defence Research and Development Organization, Government of India, New Delhi, India for financial support (ERIP/ER/DG-MSS/990516601/M/01), and Orbitron and Analytical Graphics for the simulation tools.

Received 16 April 2019; revised accepted 15 September 2020

doi: $10.18520 / \mathrm{cs} / \mathrm{v} 119 / \mathrm{i} 11 / 1774-1782$ 